\title{
Removal of Dominant Adrenal Lateralized by Glucagon-Stimulated Adrenal Venous Sampling Alleviates Hypertension in Bilateral Pheochromocytoma
}

\author{
Chandy Lou P. Malong ${ }^{1}$, Mary Jane Tanchee-Ngo ${ }^{1}$, Pilar Torres-Salvador ${ }^{1}$, Karel Pacak $^{2}$ and Leilani B. \\ Mercado-Asis ${ }^{1}$ \\ 1. Section of Endocrinology and Metabolism, Department of Medicine, University of Santo Tomas Hospital, Manila 1015, \\ Philippines
}

2. Section of Medical Neuroendocrinology, Reproductive and Adult Endocrinology Program, NICHD (National Institute of Child Health and Human Development), NIH (National Institutes of Health), Bethesda 20892-1109, Maryland, USA

Received: February 16, 2013 / Accepted: April 09, 2013 / Published: June 30, 2013.

\begin{abstract}
In bilateral pheochromocytoma, localization of the dominant adrenal is challenging but highly important since the removal of dominant side can markedly improve cardiovascular outcomes. To demonstrate the usefulness of glucagon-stimulated BAVS (bilateral adrenal venous sampling) in determining the dominant adrenal to be removed, the authors reviewed records of patients who underwent BAVS with glucagon stimulation from 1997-2010. Nineteen out of 44 patients were diagnosed with bilateral pheochromocytoma. Mean age at diagnosis was $33 \pm 14$ years. Duration of hypertension was $5 \pm 6$ years with highest systolic BP (blood pressure) of $186 \pm 30 \mathrm{mmHg}$ and diastolic BP of $113 \pm 18 \mathrm{mmHg}$. Headache (68\%) is the most common symptom followed by paroxysmal hypertension (58\%). Majority were taking $\geq 3$ anti-hypertensive drugs. On glucagon-stimulated BAVS, 63\% had right adrenal dominance. Three patients, who were hypertensive for 1, 6 and 12 years, underwent removal of the dominant adrenal. On follow-up (mean period = 36 months), there was marked improvement in BP control [pre-op vs. post-op: (systolic) 160-240 mmHg vs. 120-150 mmHg; (diastolic) $90-110 \mathrm{mmHg}$ vs. 70-90 $\mathrm{mmHg}$ ] and reduction in number of anti-hypertensive medications (from 3-5 to 2 classes of drugs). BAVS with glucagon stimulation is a valuable tool in the identification of the dominant adrenal to be removed in patients with bilateral pheochromocytoma to alleviate chronic hypertension.
\end{abstract}

Key words: Adrenal sampling, endocrine hypertension, bilateral pheochromocytoma, neuroendocrine tumor, adrenalectomy.

\section{Introduction}

The protean manifestations of PHEO (pheochromocytoma) are dependent on the quantity of catecholamines secreted by the tumor. The increased production and release of these hormones may be sustained or intermittent. There is no absolute clinical, imaging, or laboratory criteria to predict the clinical course of pheochromocytoma [1]. The diagnosis is biochemically confirmed by finding increased urinary excretion of vanillylmandelic acid, norepinephrine and

Corresponding author: Chandy Lou P. Malong, M.D., internist-endocrinologist, research fields: medicine, endocrinology. E-mail: chandyloumalong@gmail.com. epinephrine and their o-methylated metabolites, metanephrine and normetanephrine [2-4]. Currently, there are no generally agreed upon guidelines about how and which biochemical tests and imaging studies should best be carried out. In several countries, practices vary among different centers, and recommendations are more often based on the availability of these biochemical tests and on institutional experience than on evidence-based medicine [5].

The type of catecholamine produced may be helpful in the localization of the tumor through the presence of specific enzymes found neuronally or 
extra-neuronally, which convert one catecholamine to another. Utilization of these fractions into ratios may help clinicians in the localization of pheochromocytoma in patients with clinical symptoms but equivocal biochemical and imaging studies. The current recommendation on the use of venous sampling is reserved for difficult cases of pheochromocytoma especially in those whose clinical manifestations are episodic, subclinical or in patients with subcentimeter tumors. BAVS (Bilateral adrenal venous sampling) with glucagon stimulation has been proven to be safe and useful in the early diagnosis of pheochromocytoma [6]. In unilateral PHEO, the minimum predictive cut-off value of EPI (epinephrine) and NE (norepinephrine) ratio of the affected vs. unaffected side was 6.8 (sensitivity: 88.9\%; specificity: $87.5 \%$ ) and 3.8 (sensitivity: $90 \%$; specificity: $81.2 \%$ ), respectively. The EPI:NE ratio on the affected side was significantly higher (4.1 vs. 1.5). In suspected bilateral pheochromocytoma, it has been reported that the EPI:NE ratio was similar on both sides (2.42 vs. 2.24) [6]. The identification of the dominantly secreting adrenal gland can be challenging but highly important, as the removal of the affected or dominant side can markedly improve blood pressure control and halt morbidity and mortality. It is the objective to demonstrate the usefulness of glucagon-stimulated BAVS in determining the dominant adrenal gland to be surgically removed.

\section{Materials and Methods}

\subsection{Patients}

The authors retrospectively reviewed records of patients who clinically presented with signs and symptoms suggestive of pheochromocytoma and underwent BAVS with glucagon stimulation from 1997-2010 at the University of Santo Tomas Hospital. Clinical profile, medical management, surgical outcomes and follow-up were recorded. Statistical means and percentages were used in data analysis.

\subsection{Bilateral Adrenal Venous Sampling with Glucagon Stimulation}

The authors have previously described the step by step method of BAVS with glucagon stimulation [6]. The first samples were sent to Specialty Laboratories Inc., Santa Monica, California, USA and then to NUH (National University Hospital) in Singapore for determination of fractionated plasma catecholamines, NE, EPI, and dopamine by HPLC (high-performance liquid chromatography). Eventually, NUH sent the samples to the Mayo Clinic, Rochester, USA. Initially, baseline samples were assayed, but eventually only stimulated samples were studied. In the stimulated samples, serum cortisol levels were also analyzed to determine catheter position and to provide a denominator to control for the relative dilution of catecholamines in samples from each side [7]. Only NE and EPI gradients were analyzed in the study.

The minimum predictive cut-off value for the EPI ratio of the affected vs. unaffected side in diagnosing unilateral pheochromocytoma was 6.8 with a sensitivity of $88.9 \%$ and specificity of $87.5 \%$. A ratio of 20.4 would yield a specificity of $100 \%$ with a sensitivity of $83.3 \%$. The minimum predictive cut-off value for the NE ratio of affected vs. unaffected sides in diagnosing unilateral pheochromocytoma was 3.8 with a sensitivity of $90 \%$ and specificity of $81.2 \%$. The EPI:NE ratio on the affected side was significantly higher (4.1 vs. 1.5). In suspected bilateral pheochromocytoma, the EPI:NE ratio was similar on both sides (2.42 vs. 2.24) [6]. Majority of patients tolerated the procedure with no untoward events.

In the 14-year period, 44 patients underwent the procedure. Five had normal results (EPI $<100 \mathrm{pg} / \mathrm{mL}$, $\mathrm{NE}<750 \mathrm{pg} / \mathrm{mL}$ ), 20 were diagnosed with unilateral pheochromocytoma, and 19 were suspicious of having bilateral pheochromocytoma. Patients were given an option to have the dominant adrenal removed. 


\section{Results}

\subsection{Demographic Profile}

Nineteen patients, 10 males and 9 females, aged 18 to 65 years (mean $38.6 \pm 13.6$ years) were diagnosed with bilateral pheochromocytoma. The mean age at diagnosis was $33.4 \pm 13.9$ years. The mean duration of hypertension was $5.2 \pm 6.4$ years with an average highest systolic BP (blood pressure) of $185.9 \pm 29.3$ mmHg and diastolic BP of $113.0 \pm 18.2 \mathrm{mmHg}$. Majority were taking three or more anti-hypertensive medications. Headache (68\%) is the most common symptom followed by paroxysmal hypertension (58\%) and palpitation (42\%) (Table 1).

Computed tomography scan of the adrenals with contrast (1-3 mm cut) was performed which showed no definite mass in any of the patients. Some patients presented for consult with elevated plasma or urine catecholamine or metanephrine levels.

\subsection{Biochemical Profile on BAVS with Glucagon Stimulation}

On glucagon-stimulated BAVS, 63\% had right

Table 1 Signs and symptoms of patients suspicious of having bilateral pheochromocytoma.

\begin{tabular}{lr}
\hline Signs and symptoms & $N(\%)$ \\
\hline Headache & $13(68.4)$ \\
Paroxysmal hypertension & $11(57.9)$ \\
Palpitation & $8(42.1)$ \\
Flushing & $7(36.8)$ \\
Anxiety & $5(26.3)$ \\
Diaphoresis & $4(21.1)$ \\
Dizziness & $3(15.8)$ \\
Weakness & $3(15.8)$ \\
\hline
\end{tabular}

adrenal dominance. The endocrine profiles of patients suspicious of having bilateral pheochromocytoma were shown in Table 2. The mean epinephrine and norepinephrine levels on the dominant side were $24,506 \pm 30,710 \mathrm{pg} / \mathrm{mL}$ and 8,642 \pm 13,395 pg/mL, respectively. The mean ratio of the dominant versus non-dominant adrenal for epinephrine and norepinephrine were 3.62 and 4.13, respectively. The degree of catecholamine hypersecretion observed in the adrenal glands was not seen in the periphery (EPI $=151 \pm 106 \mathrm{pg} / \mathrm{mL}$; NE= $392 \pm 261 \mathrm{pg} / \mathrm{mL}$ ).

In the three cases, glucagon-stimulated metanephrines and normetanephrines were measured during adrenal sampling, in addition to the epinephrine and norepinephrine levels (Table 3). The absolute values of the o-methylated metabolites were higher than the catecholamines. Likewise, the metanephrine to normetanephrine ratio was relatively high as compared to epinephrine to norepinephrine ratio. However, cut-off values for metanephrine and normetanephrine ratios are not yet established.

\subsection{Outcome after Surgery}

Of the 19 patients with bilateral PHEO, only three patients, who were hypertensive for 1, 6 and 12 years, underwent removal of the dominant adrenal. On follow-up (mean period $=36$ months), there was marked improvement in blood pressure control regardless of the duration of hypertension (Table 4).

There was also a reduction in the number of anti-hypertensive medications from 3-5 to 2 classes of drugs.

Table 2 Glucagon stimulated epinephrine and norepinephrine levels in patients with suspected bilateral pheochromocytoma.

\begin{tabular}{|c|c|c|c|}
\hline \multirow{2}{*}{ Catecholamine } & \multicolumn{2}{|c|}{ Suspected bilateral tumors } & \multirow{2}{*}{ - Ratio } \\
\hline & Dominant $^{\mathrm{a}}$ side & Non-dominant ${ }^{\mathrm{b}}$ side & \\
\hline Epinephrine (mean \pm SD) & $24,506 \pm 30,710 \mathrm{pg} / \mathrm{mL}$ & $12,444 \pm 18,720 \mathrm{pg} / \mathrm{mL}$ & 3.62 \\
\hline Norepinephrine (mean \pm SD) & $8,642 \pm 13,395 \mathrm{pg} / \mathrm{mL}$ & $3,829 \pm 7,137 \mathrm{pg} / \mathrm{mL}$ & 4.13 \\
\hline Ratio of EPI:NE ${ }^{\mathrm{c}}$ & 3.4 & 3.2 & \\
\hline
\end{tabular}

${ }^{\mathrm{a} D o m i n a n t: ~ t h e ~ a d r e n a l ~ s i d e ~ w i t h ~ t h e ~ h i g h e r ~ g l u c a g o n-s t i m u l a t e d ~ r e s p o n s e ; ~}{ }^{\mathrm{b}}$ Non-dominant: the adrenal side with the lower glucagon-stimulated response; ${ }^{\mathrm{C} A b b r e v i a t i o n s: ~ E P I: ~ e p i n e p h r i n e ; ~ N E: ~ n o r e p i n e p h r i n e . ~}$ 
Table 3 Stimulated fractionated catecholamine levels by bilateral adrenal venous sampling with glucagon stimulation.

\begin{tabular}{|c|c|c|c|}
\hline BAVS with glucagon stimulation & Case I & Case II & Case III \\
\hline \multicolumn{4}{|l|}{ Dominant adrenal gland } \\
\hline Epinephrine (pg/mL) & 154 & 1,886 & $>17,500$ \\
\hline Norepinephrine (pg/mL) & 31 & 1,263 & 7,660 \\
\hline Metanephrine (pg/mL) & 4,975 & 5,080 & $>2,000$ \\
\hline Normetanephrine (pg/mL) & 935 & 1,845 & Immeasurably high \\
\hline \multicolumn{4}{|l|}{ Non-dominant adrenal gland } \\
\hline Epinephrine (pg/mL) & 86 & 785 & 14,962 \\
\hline Norepinephrine (pg/mL) & 26 & 650 & 4,513 \\
\hline Metanephrine (pg/mL) & 3,421 & 1,473 & $>2,000$ \\
\hline Normetanephrine (pg/mL) & 649 & 551 & Immeasurably high \\
\hline \multicolumn{4}{|l|}{ Dominant vs. non-dominant } \\
\hline Epinephrine ratio & 1.80 & 2.40 & 1.16 \\
\hline Metanephrine ratio & 1.45 & 3.44 & 1.0 \\
\hline Norepinephrine ratio & 1.20 & 1.94 & 1.7 \\
\hline Normetanephrine ratio & 1.44 & 3.34 & Immeasurably high \\
\hline \multicolumn{4}{|l|}{ Epinephrine:Norepinephrine ratio } \\
\hline Dominant & 4.97 & 1.5 & 2.28 \\
\hline Non-dominant & 3.3 & 1.2 & 3.31 \\
\hline \multicolumn{4}{|c|}{ Metanephrine:Normetanephrine ratio } \\
\hline Dominant & 5.32 & 2.75 & Immeasurably high \\
\hline Non-dominant & 5.27 & 2.67 & Immeasurably high \\
\hline
\end{tabular}

Table 4 Outcomes of three patients who underwent surgical removal of the dominant adrenal.

\begin{tabular}{|c|c|c|c|c|c|c|c|c|}
\hline \multirow{3}{*}{ Case } & \multirow{3}{*}{$\begin{array}{l}\text { Duration of } \\
\text { hypertension } \\
\text { (years) }\end{array}$} & \multirow{3}{*}{$\begin{array}{l}\text { Follow-up } \\
\text { period } \\
\text { (month) }\end{array}$} & \multicolumn{4}{|c|}{$\mathrm{BP}(\mathrm{mmHg})$} & \multirow{2}{*}{\multicolumn{2}{|c|}{ Anti-hypertensive medications $(N)$}} \\
\hline & & & \multicolumn{2}{|c|}{ Systolic } & \multicolumn{2}{|c|}{ Diastolic } & & \\
\hline & & & Pre-op & Post-op & Pre-op & Post-op & Pre-op & Post-op \\
\hline 1 & 12 & 24 & $180-240$ & $130-140$ & $100-110$ & 2 & 5 & \\
\hline 2 & 6 & 36 & $160-200$ & $120-150$ & $90-100$ & 2 & 4 & \\
\hline 3 & 1 & 49 & $160-180$ & $120-150$ & $90-100$ & $\cdot 2$ & 3 & \\
\hline
\end{tabular}

\section{Discussion}

The current recommendation on the use of venous sampling is reserved for difficult cases of pheochromocytoma. Selective venous catheterization and the assay of plasma catecholamines should be considered for localization in: (1) patients in whom standard techniques fail to localize the tumor; (2) patients who exhibit idiosyncratic reactions to the angiographic contrast materials; (3) young patients or patients with familial pheochromocytoma, including those with multiple neurofibromatosis or multiple endocrine adenomatosis, type 2; (4) patients with recurrent, malignant, or suspected multicentric or extra-adrenal tumours; (5) patients excreting only norepinephrine in the urine. The validity of the results is particularly dependent on the skill with which venous catheterization is carried out. Coupled with measurement of catecholamines or, preferably, metanephrines to localize the tumor through the discovery of a secretory gradient, it is an ultimate modality to be used with caution in selected cases where all imaging methods have failed [1, 8].

The production of metanephrines varies widely among tissues from locally released and circulating catecholamines. The metabolism of circulating norepinephrine was responsible for only $19 \%$ of the total production of normetanephrine (NMN) and an even smaller portion (6\%) of plasma metanephrine (MN) was derived from metabolism of circulating 
epinephrine. Most plasma MN (91\%) was produced within the adrenals, which also provided the largest single source (23\%) of NMN. The substantial contribution of the adrenals to the production of MNs explains the extraordinary sensitivity of these metabolites for the diagnosis of pheochromocytoma [9]. However, the high incidence of false-positive tests remains a problem.

By far, the use of the glucagon test to establish a positive result depends on demonstration of a large increment in plasma concentrations of norepinephrine within $3 \mathrm{~min}$ of an intravenous bolus injection of glucagon. This evoked release appears dependent on the presence of the glucagon receptor. Inadequate response characterizes negative test results in patients without pheochromocytoma [10]. The test, however, is fraught with numerous methodological problems and the lack of adequately defined criteria for establishing positive and negative test results from responses of both NE and EPI. Several of the studies have used a relatively small number of population [11-15]. It is reported by the group of lenders that the pheochromocytoma-associated percentage elevations in median baseline plasma concentrations of normetanephrine and metanephrine were larger than respective elevations of norepinephrine and epinephrine. Median baseline plasma concentrations of normetanephrine in patients with pheochromocytoma were $620 \%$ to $660 \%$ higher than in subjects without the tumor (compared with only $180 \%$ to $360 \%$ for norepinephrine). Plasma concentrations of metanephrine were $70 \%$ to $190 \%$ higher than in subjects without the tumor (compared with $60 \%$ to $90 \%$ for epinephrine). Though glucagon provocative testing has insufficient diagnostic sensitivity, the highest diagnostic specificity of $100 \%$ is achieved if increases in plasma concentrations of norepinephrine were more than 3-fold [16]. Catecholamine levels in these studies were extracted from the peripheral vessels. It is of note that the samples in our series of patients were obtained via adrenal sampling which is more reflective of the true secretion of these hormones from adrenal source and improves the sensitivity of the test. The degree of catecholamine hypersecretion observed in the adrenal glands was also not seen in the peripheral samples in our series.

All cases presented are confounded by the inconclusive biochemical test results and equivocal imaging studies. The use of adrenal venous sampling to identify the dominant lesion, combined with the improved sensitivity of plasma catecholamine measurements stimulated by glucagon proved to be a valuable diagnostic tool. The removal of the lesion with higher epinephrine and norepinephrine secretions in the three cases has resulted in clinical improvement, reduction in blood pressure and number of anti-hypertensive medications. Thus, this can prevent long term fatal cardiovascular complications.

\section{Conclusion}

In conclusion, the authors have demonstrated the usefulness of glucagon-stimulated BAVS in determining the dominant adrenal gland to be surgically removed to alleviate hypertension. Authors have likewise presented the first 3 cases where glucagon stimulated metanephrines and normetanephrines were measured during adrenal sampling. Further studies involving higher number of patients to establish the sensitivity and specificity of this procedure in the diagnosis and localization of small and bilateral pheochromocytoma are recommended.

\section{Acknowledgments}

This work was accomplished through review of patient records and thus, no funding was necessitated. It is with the combined support and generosity of the Section of Endocrinology and Metabolism of the University of Santo Tomas Hospital and the National Institutes of Health that this manuscript was completed. 


\section{Removal of Dominant Adrenal Lateralized by Glucagon-Stimulated Adrenal Venous Sampling Alleviates Hypertension in Bilateral Pheochromocytoma}

\section{References}

[1] I. Ilias, K. Pacak, Current approach and recommended algorithm for the diagnostic localization of pheochromocytoma, J Clin Endocrinol Metab. 89 (2) (2004) 479-491.

[2] G. Eisenhofer, D.S. Goldstein, M.M. Wather, P. Friberg, J. Lenders, H. Keiser, et al., Biochemical diagnosis of pheochromocytoma: How to distinguish true-from false-positive test results, J Clin Endocrinol Metab. 88 (6) (2003) 2656-2666.

[3] E. Bravo, R. Tagle, Pheochromocytoma: State-of-the-art and future prospects, Endocr Rev. 24 (4) (2003) 539-553.

[4] Y. Kudva, A. Sawka, W. Young, The laboratory diagnosis of adrenal pheochromocytoma: The mayo clinic experience, J Clin Endocrinol Metab. 88 (10) (2003) 4533-4539.

[5] A. Grossman, K. Pacak, A. Sawka, J. Lenders, D. Harlander, R. Peaston, et al., Biochemical diagnosis and localization of pheochromocytoma: Can we reach a consensus?, Ann N.Y. Acad Sci. 1073 (2006) 332-347.

[6] L.B. Mercado-Asis, A. Tingcungco, D. Bolong, R. Lopez, E. Caguioa, M. Yamamoto, et al., Diagnosis of small adrenal pheochromocytomas by adrenal venous sampling with glucagon stimulation test, Int J Endocriol Metab. 2 (2) (2011) 323-329.

[7] N.A. Samaan, R.C. Hickey, P.E. Shutts, Diagnosis, localization, and management of pheochromocytoma. Pitfalls and follow-up in 41 patients, Cancer 62 (11) (1988) 2451-2460.

[8] K. Pacak, D. Goldstein, J. Doppman, B. Shulkin, R. Udelsman, G. Eisenhofer, A "Pheo" lurks: Novel approaches for locating occult pheochromocytoma, J Clin
Endocrinol Metab 86 (8) (2001) 3641-3646.

[9] V. Fiorica, D.C. Galloway, J.L. Males, R. Painton, D. Kem, Epinephrine fraction of adrenal vein blood in pheochromocytoma, Am J Med Sci. 284 (3) (1982) 9-15.

[10] G.S. Levey, S.R. Weiss, E. Ruiz, Characterization of the glucagon receptor in a pheochromocytoma, J Clin Endocrinol Metab. 40 (4) (1975) 720-723.

[11] S.G. Sheps, F.T. Maher, Histamine and glucagon tests in diagnosis of pheochromocytoma, JAMA 205 (13) (1968) 895-859.

[12] E.L. Bravo, R.C. Tarazi, R.W. Gifford, B.H. Stewart, Circulating and urinary catecholamines in pheochromocytoma: Diagnostic and pathophysiologic implications, N Engl J Med. 301 (13) (1979) 682-686.

[13] G.P. Bernini, M.S. Vivaldi, G.F. Argenio, A. Moretti, M. Sgro, A. Salvetti, Frequency of pheochromocytoma in adrenal incidentalomas and utility of the glucagon test for the diagnosis, J Endocrinol Invest. 20 (2) (1997) 65-71.

[14] P.D. Levinson, B.P. Hamilton, J.H. Mersey, A.A. Kowarski, Plasma normetanephrine and epinephrine responses to glucagon in patients with suspected pheochromocytomas, Metabolism 32 (10) (1983) 998-1001.

[15] E. Grossman, D.S. Goldstein, A. Hoffman, H. Keiser, Glucagon and clonidine testing in the diagnosis of pheochromocytoma, Hypertension 17 (6 pt 1) (1991) 733-741.

[16] W.M. Lenders, K. Pacak, T. Huynh, Y. Sharabi, M. Mannelli, G. Bratslavsky, et al., Low sensitivity of glucagon provocative testing for diagnosis of pheochromocytoma, J Clinical Endocrinol Metab. 95 (1) (2010) 238-245 